\title{
COMPARISON OF 2D AND 3D COMPUTATIONAL MULTIPHASE FLUID FLOW MODELS OF OXYGEN LANCING OF PYROMETALLURGICAL FURNACE TAP-HOLES
}

\author{
M.W. Erwee ${ }^{1,2}$, Q.G. Reynolds ${ }^{1}$ and J.H. Zietsman ${ }^{2}$ \\ ${ }^{1}$ Mintek, 200 Malibongwe Drive, Randburg, Gauteng, 2194, South Africa, \\ markuse@mintek.co.za; \\ ${ }^{2}$ University of Pretoria, Lynnwood Road, Pretoria, Gauteng, 0002, South Africa
}

\section{Abstract}

Furnace tap-holes vary in design depending on the type of furnace and process involved, but share one common trait: the tap-hole must be opened and closed periodically. In general tapholes are plugged with refractory clay after tapping, thereby stopping the flow of molten material. Once a furnace is ready to be tapped, drilling and/or lancing with oxygen are typically used to remove tap-hole clay from the tap-hole. Lancing with oxygen is an energy-intensive, mostly-manual process, which affects the performance and longevity of the tap-hole refractory material as well as the processes inside the furnace. Computational modeling offers an opportunity to gain insight into the possible effects of oxygen lancing on various aspects of furnace operation.

\section{Introduction}

In pyrometallurgical processes, molten material resulting from smelting must be removed from the furnace in some way. In most cases the furnace is tapped through one or more tap-holes situated around the furnace. Furnace tap-holes differ in design depending on the type of furnace and process involved, but for the most part share one important feature: the need to open and close the tap-hole at different stages of the pyrometallurgical process.

A furnace tap-hole is usually a channel passing through a specialized refractory assemblage. Tap-hole refractories are specially designed to withstand high temperatures, thermal shock, and erosion (chemical and mechanical) by molten material flowing through them. After tapping, the tap-hole is closed using what is commonly referred to as a 'mud-gun', a hydraulic device that injects refractory clay into the tap-hole with a significant pressure. The clay expands and hardens inside the tap-hole, effectively closing it. Once the furnace is to be tapped again, the refractory plug is removed by drilling and/or lancing into the hardened clay.

Drilling is generally performed using a semi-automated pneumatic drill. In most cases, however, it is not possible to drill completely through the clay plug to open the tap-hole, and oxygen lancing is required to complete the process. The extent to which lancing forms part of opening a tap-hole is largely based on the type of furnace used for processing. In blast furnaces [1], drilling is preferred and oxygen lancing is used less 
than $1 \%$ of the time, while in platinum smelting lancing is used much more frequently (up to a third of the time [2]).

Oxygen lancing is, for the most part, a manual process (see Figure 1), although automated lancing systems are becoming a reality. A lance is usually a steel pipe (various designs exist) connected to an oxygen tank or oxygen gas line. The oxygen flows through the pipe to the tip of the lance where it is used to oxidise the iron in the steel and release thermal energy. The tip of the lance hence serves as a concentrated source of heat which is used to melt away the refractory clay in the furnace tap-hole, penetrating the furnace and allowing molten material to be tapped.

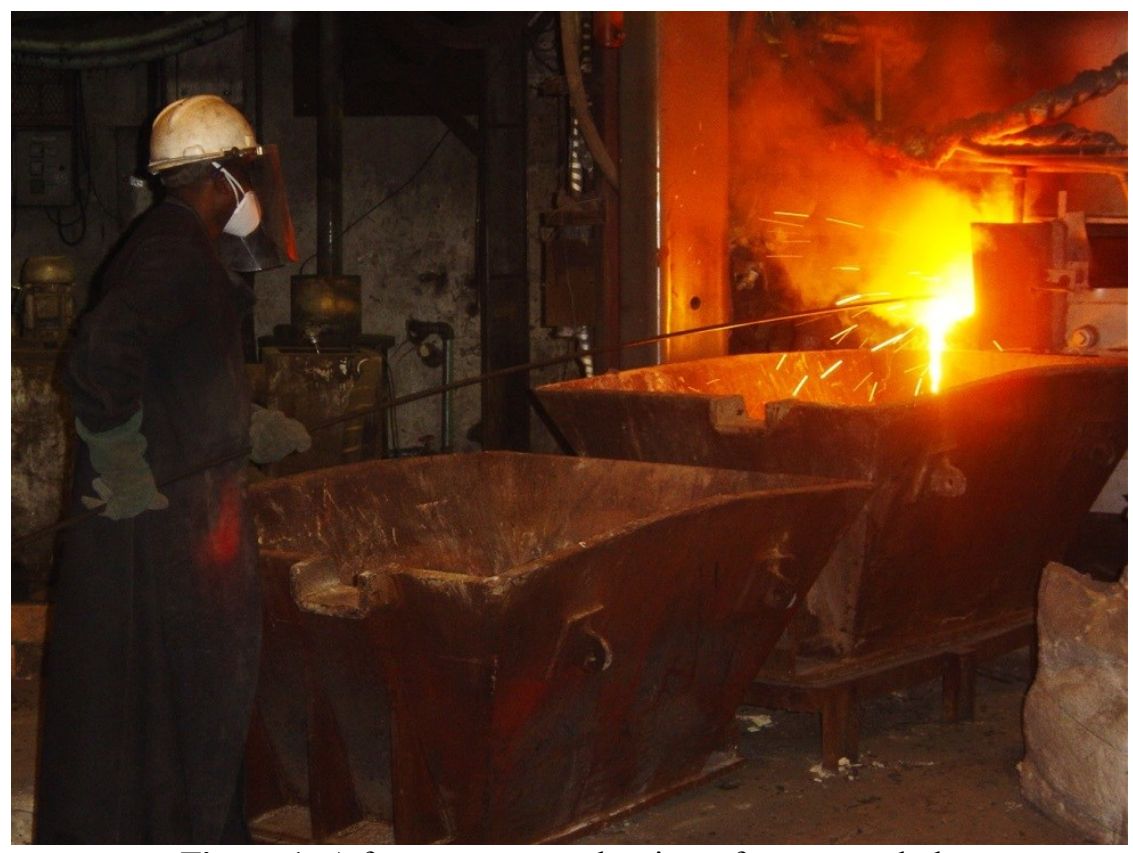

Figure 1: A furnace operator lancing a furnace tap-hole on a $200 \mathrm{kVA}$ DC furnace at Mintek.

(Photograph: IJ Geldenhuys, Mintek, 2004)

\subsection{Purpose of this work}

Oxygen lancing is a highly energy-intensive and aggressive process, and as such it affects the life and integrity of a tap-hole. Experience at Mintek together with anecdotal evidence from many industrial plants has indicated that the lancing procedure, type of lance, and other factors associated with the oxygen lancing process affect the performance of the tap-hole. Aside from the longevity of the tap-hole and surrounding area, introducing oxygen into a furnace through the tap-hole may also cause local disturbances in the molten material in the vicinity of the tap-hole due to multiphase fluid flow phenomena as well as reaction between oxygen and the material inside the furnace (slag or metal). To this end, this study aims to quantify through computational modeling and experimental validation the possible effects of oxygen lancing on the process and subsequently on the tap-hole and tap-block assembly.

Previous work by the same authors [3] aimed to validate a two-dimensional computational multiphase fluid flow model against an experimental water-based model. In the present work, the 
two-dimensional model was expanded to three dimensions in order to address some of the shortcomings identified in [3].

\subsection{Short summary of previous work done}

In the previous set of work [3], the focus was on comparing a two-dimensional multiphase computational fluid flow model against an experimental model. A simple rectangular tank was constructed from polymethylmetacrylate (Plexiglass ${ }^{\circledR}$ or Perspex ${ }^{\circledR}$ ) with dimensions $0.2 \mathrm{~m}$ high, $0.4 \mathrm{~m}$ wide and $0.045 \mathrm{~m}$ deep. The tank was filled with water to a level of $0.15 \mathrm{~m}$. A copper tube (internal diameter $2.91 \mathrm{~mm}$ ) was used as a lance analogue. The lance was positioned in the tank at different positions along the width of the tank. The flow rate of gas through the lance was also varied.

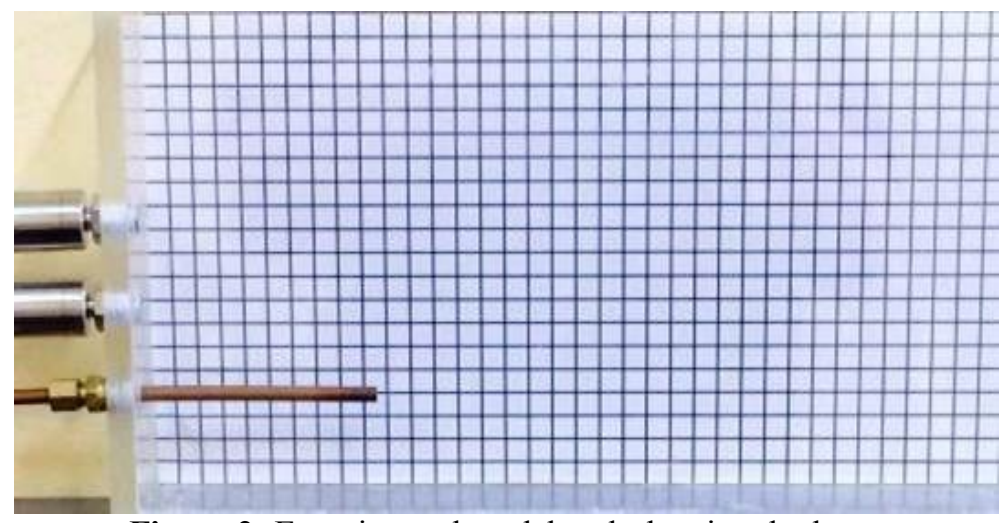

Figure 2: Experimental model tank showing the lance (copper tube at bottom left), with two pressure sensors positioned above the lance at different positions (see reference [3] for more detail).

Pressure sensors (IPSL type from RS Components, range: 0-50 mbar gauge pressure) were used to record pressure signals on the wall of the tank at two different locations. An Olympus iSpeed 3 high-speed video camera was used to record the experimental work. The pressure signals were then compared to predicted pressure signals generated from computational models of the system. High-speed video footage of the experiment was also compared visually against the computational model results.

Substantial differences between the mean pressure measurements and modelled mean pressure were observed, but there was generally good agreement in the pressure standard deviation, which was used as an indicator of the magnitude of fluctuations in the pressure signal. It was suggested that the computational multiphase fluid flow model be extended to three dimensions to confirm this result. It was also suggested that the sampling rate of the experimentally-measured pressure signal be increased to more closely match the sampling rates available in the computational models. It was also suggested that the pressure signals be analysed in more detail using Fourier analysis.

Visual comparison of the high-speed video footage to the predictions of the computational model also showed reasonable agreement with respect to bulk behaviour of the fluids, although significantly exaggerated splashing was predicted in the computational model compared to what 
was observed experimentally; this was an additional argument for extending the computational model to a three dimensions.

\section{Computational multiphase fluid flow model}

\subsection{Governing equations}

Three main equations are required for the solution of multiphase fluid flow problems using the volume-of-fluid method [7].

The first is the Navier-Stokes equation for conservation of momentum:

$$
\frac{\partial(\rho \boldsymbol{u})}{d t}+\nabla \cdot(\rho \boldsymbol{u u})+\nabla P=\nabla \cdot \tau_{i j}-\left[\gamma \nabla \cdot\left(\frac{\nabla \alpha}{|\nabla \alpha|}\right)\right] \nabla \alpha+\rho \boldsymbol{g}
$$

Where $\mathbf{u}$ is the velocity vector, $P$ is the pressure, $\rho$ is density, $\tau_{\mathrm{ij}}$ is the viscous shear force term, and $\gamma$ is surface tension (all physical properties are interpolated linearly in the models). The term $\alpha$ is the phase fraction of the primary phase (in this case $\alpha=1$ for water, while $\alpha=0$ for air).

The other two equations to be solved are the continuity equations, which express the conservation of mass both overall and per phase:

$$
\begin{aligned}
& \frac{\partial \rho}{d t}+\nabla \cdot(\rho \boldsymbol{u})=0 \\
& \frac{\partial \alpha}{d t}+\nabla \cdot(\alpha \boldsymbol{u})=0
\end{aligned}
$$

\subsection{Modeling framework and solvers}

OpenFOAM ${ }^{\circledR}[4]$, an open-source framework for field solution of the conservation equations, was chosen as the computational modeling platform to implement the computational multiphase fluid flow model. Meshing was performed using the open-source platform Gmsh [5]. The standard interFoam solver for two-phase incompressible flow in OpenFOAM ${ }^{\circledR}$ was used. The solver consists of a flow model and a phase separation model. Turbulence modeling was not considered as the lance injection velocities are relatively low and the aim was to use sufficiently high mesh resolutions to fully resolve the flow.

OpenFOAM ${ }^{\circledR}$ discretizes the Navier-Stokes, continuity, and phase conservation equations governing the different fields using the finite-volume method [6].

The VOF method requires the solution of a separate convective transport equation for the volume fraction field of the primary phase, $\alpha$. An attractive feature of the VOF method is that the need for computationally-expensive explicit interface tracking and moving mesh calculations is entirely avoided. 
The velocity and pressure fields at each time step were calculated using the Pressure Implicit with Splitting of Operators predictor-corrector (PISO) algorithm. Gradient-limited discretisations were used for the divergence terms, except for those related to the phase fraction field, for which the Multidimensional Universal Limiter for Explicit Solution (MULES) limiter was used to perform interface compression and capturing.

To ensure numerical stability of the algorithm, a Courant number of 1 or below was maintained using adaptive time-stepping.

\subsection{Boundary Conditions}

The walls of the lance and vessel were treated as non-slip, non-permeable boundaries with velocity $=0$, normal gradient of pressure $=0$ and normal gradient of $\alpha=0$. The top surface of the model was assumed to be open to atmosphere, with a fixed gauge pressure $=0$, velocity calculated from the pressure gradient, and an inlet-outlet boundary condition for $\alpha$ based on the direction of flow across the boundary ( $\alpha=0$ (air) if inflow, normal gradient of $\alpha=0$ if outflow). At the lance tip, a fixed constant velocity in the horizontal direction was specified (see section 5.4), together with the normal gradient of pressure $=0$, and a fixed value of $\alpha=0$.

\subsection{Scenarios considered}

Previously, a two-dimensional slice model of the water tank system was developed [3]. For the present work, the same model was expanded to a three-dimensional model. There were slight differences in the versions of software used for meshing as well as solving the models (see Table I). The three-dimensional models required substantially more computing power to solve the models within a reasonable timeframe.

For both the two- and three-dimensional models, the same variables were studied. These include the lance penetration depth, as well as the gas flow rate. The depth of the lance was varied between $1 \mathrm{~cm}, 5 \mathrm{~cm}$ and $10 \mathrm{~cm}$ from the wall where the lance enters along the width of the tank, while the gas flow rate was set to $1.0,3.5$ or $7.5 \mathrm{~L} / \mathrm{min}$ at each depth setting.

\subsection{Meshing and computational requirements}

For the two-dimensional model, unstructured quadrilateral elements were used (hexahedral mesh reduced to two dimensions). Polyhedral meshes were created for the three-dimensional model. A complication arose in generating a three-dimensional mesh: concave curves (in this case the tip of the lance) were found to be difficult to mesh with polyhedral elements using the selected software tools. The tip of the lance was slightly chamfered in the model to counter this effect. The mesh was refined in the regions against the wall and around the lance tip to ensure that pressure fluctuations could be accurately captured.

Examples of the meshes used by the two- and three-dimensional models are shown in Figure 3 and Figure 4. 


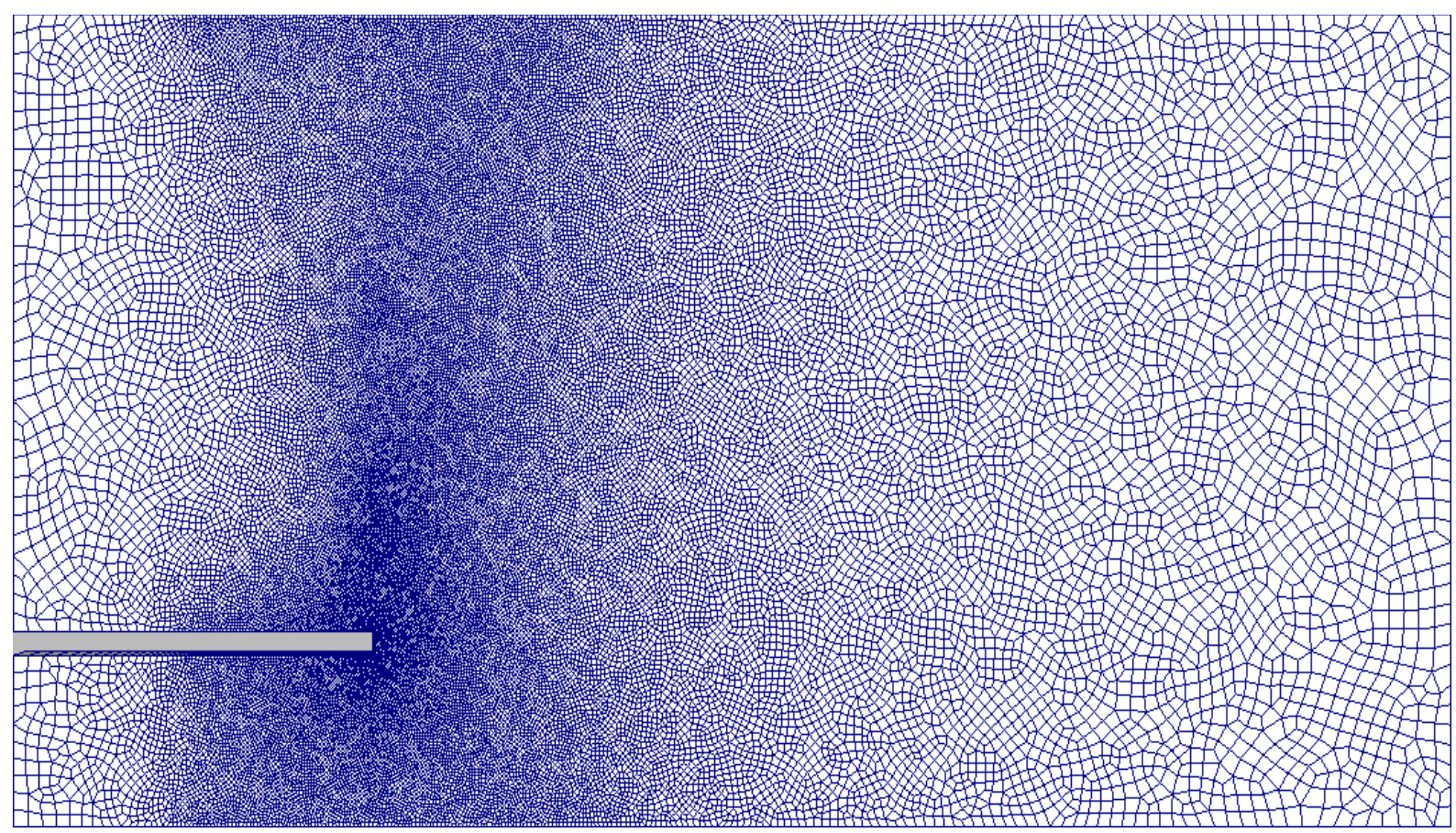

Figure 3: A typical two-dimensional mesh for a lance depth of $10 \mathrm{~cm}$ (40218 elements)

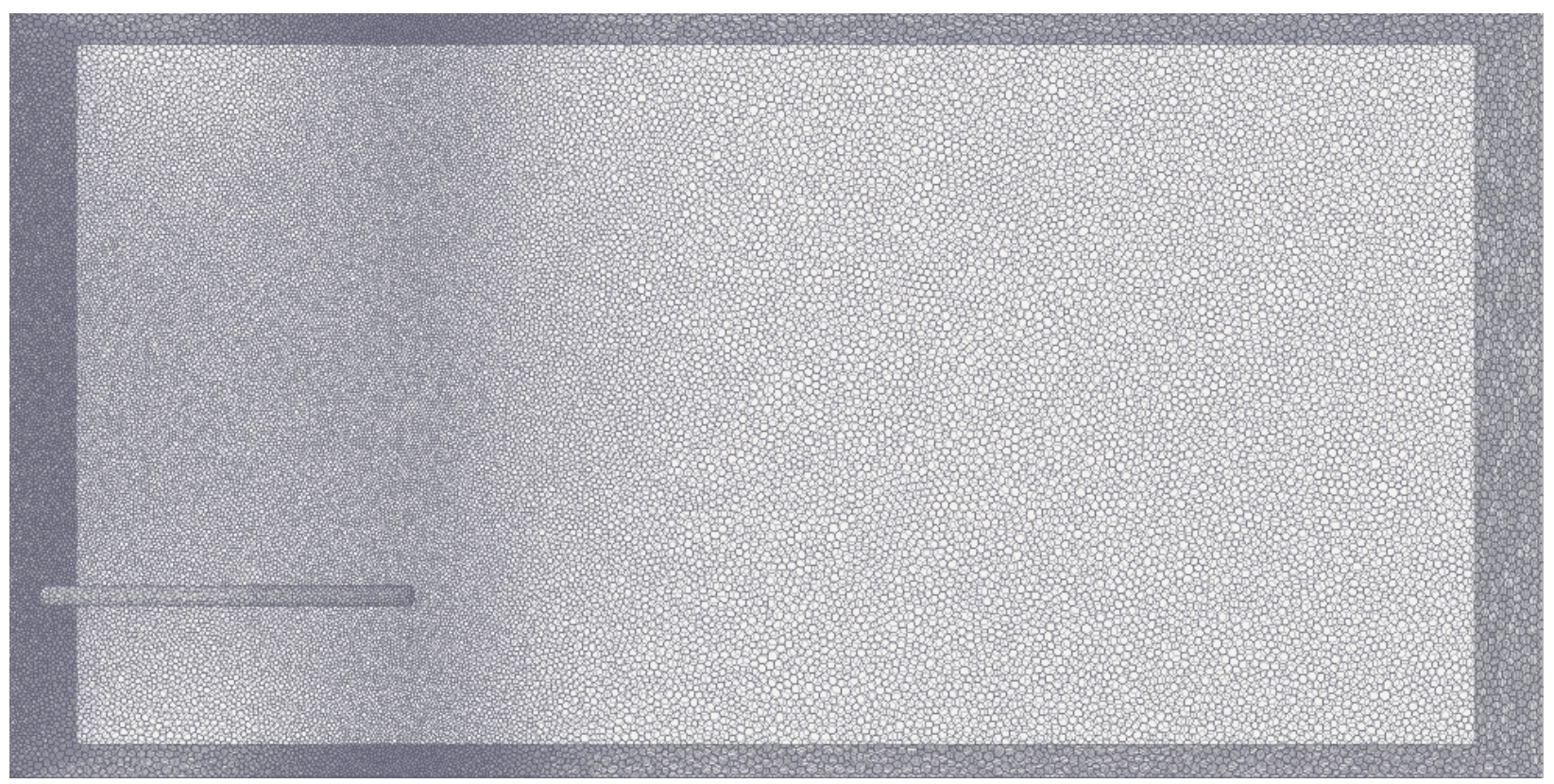

Figure 4: A typical three-dimensional mesh for a lance depth of $10 \mathrm{~cm}$ (656205 polyhedral elements)

The software packages used, along with the versions used for the models are shown in Table I. 
Table I: Software versions used for modeling

\begin{tabular}{|l|c|c|}
\hline Software & 2D Computational Model & 3D Computational Model \\
\hline OpenFOAM $\mathbb{R}$ Version & 2.3 .1 & 2.4 .0 \\
\hline Solver & \multicolumn{2}{|c|}{ interFoam } \\
\hline Mesh type & Quadrilateral & Polyhedral \\
\hline Meshing tool & GMSH 2.0.3 & $\begin{array}{c}\text { GMSH 2.1.1; convert from } \\
\text { tetrahedral to polyhedral using } \\
\text { polyDualMesh (OF) }\end{array}$ \\
\hline
\end{tabular}

The scenarios run, as well as the computing resources used to run the different models are shown in Table II. The two-dimensional models were at least an order of magnitude smaller in terms of mesh size than the three-dimensional models. This is a very important consideration when modeling large furnaces. The two-dimensional models were solved on a small Linux cluster at Mintek, while the three-dimensional models were solved using resources at the Centre for High Performance Computing in Cape Town, South Africa.

Table II: Scenarios, sizes of meshes and computing power used for modeling

\begin{tabular}{|c|c|c|c|c|c|c|c|}
\hline \multirow[t]{2}{*}{$\begin{array}{l}\text { Lance Depth } \\
\text { (cm into tank) }\end{array}$} & \multirow[t]{2}{*}{$\begin{array}{c}\text { Gas Flow Rate } \\
\text { (L/min) }\end{array}$} & \multicolumn{2}{|c|}{$\begin{array}{c}\text { Number of } \\
\text { elements in mesh }\end{array}$} & \multicolumn{2}{|c|}{$\begin{array}{l}\text { Size of coarsest } \\
\text { element in mesh } \\
\text { (m) }\end{array}$} & \multicolumn{2}{|c|}{ No of cores used } \\
\hline & & $2 \mathrm{D}$ & $3 \mathrm{D}$ & $2 \mathrm{D}$ & $3 \mathrm{D}$ & $2 \mathrm{D}$ & $3 \mathrm{D}$ \\
\hline \multirow{3}{*}{10.0} & 1.0 & \multirow{3}{*}{40218} & \multirow{3}{*}{656205} & \multirow{11}{*}{0.001} & \multirow{3}{*}{0.0025} & \multirow{9}{*}{$\begin{array}{c}4 \\
(1: 4: 8)^{1}\end{array}$} & \multirow{9}{*}{$\begin{array}{c}16 \\
(2: 8: 8)\end{array}$} \\
\hline & 3.5 & & & & & & \\
\hline & 7.5 & & & & & & \\
\hline \multirow{3}{*}{5.0} & 1.0 & \multirow{3}{*}{30861} & \multirow{3}{*}{440766} & & \multirow{6}{*}{0.005} & & \\
\hline & 3.5 & & & & & & \\
\hline & 7.5 & & & & & & \\
\hline \multirow{3}{*}{1.0} & 1.0 & \multirow{3}{*}{19423} & \multirow{3}{*}{278118} & & & & \\
\hline & 3.5 & & & & & & \\
\hline & 7.5 & & & & & & \\
\hline 10.0 & 7.5 & \multirow{2}{*}{ - } & 2815263 & & \multirow{2}{*}{0.001} & \multirow{2}{*}{-} & \multirow{2}{*}{$\begin{array}{c}64 \\
(8: 8: 8)\end{array}$} \\
\hline 1.0 & 7.5 & & 2824939 & & & & \\
\hline
\end{tabular}

\subsection{Results}

\subsubsection{Means of comparison}

In this paper, the two computational models (2D and 3D) are qualitatively and quantitatively compared. A significant part of modelling work, especially in three dimensions, is to study how dependent the results are on mesh resolution. Higher mesh resolutions generally produce better accuracy, but come at increased computational cost - a trade-off between accuracy and performance is therefore always required.

\footnotetext{
${ }^{1}$ (Number of Nodes: Number of Cores per Node: Number of MPI Processes)
} 
In Figure 5, the cost of running the models is shown. At very high mesh resolutions (above 1 million elements), even with four times the computing power of those with reasonable resolutions (less than 1 million elements), the models require roughly double the time to run. It was found that running simulations using the 3D model still showed differences in pressure fluctuations predicted after the mesh was refined from 656205 elements (for a lance depth of 10 $\mathrm{cm})$ to 2815263 elements. Mesh independence for this problem is still being studied, and will be published at a later stage.

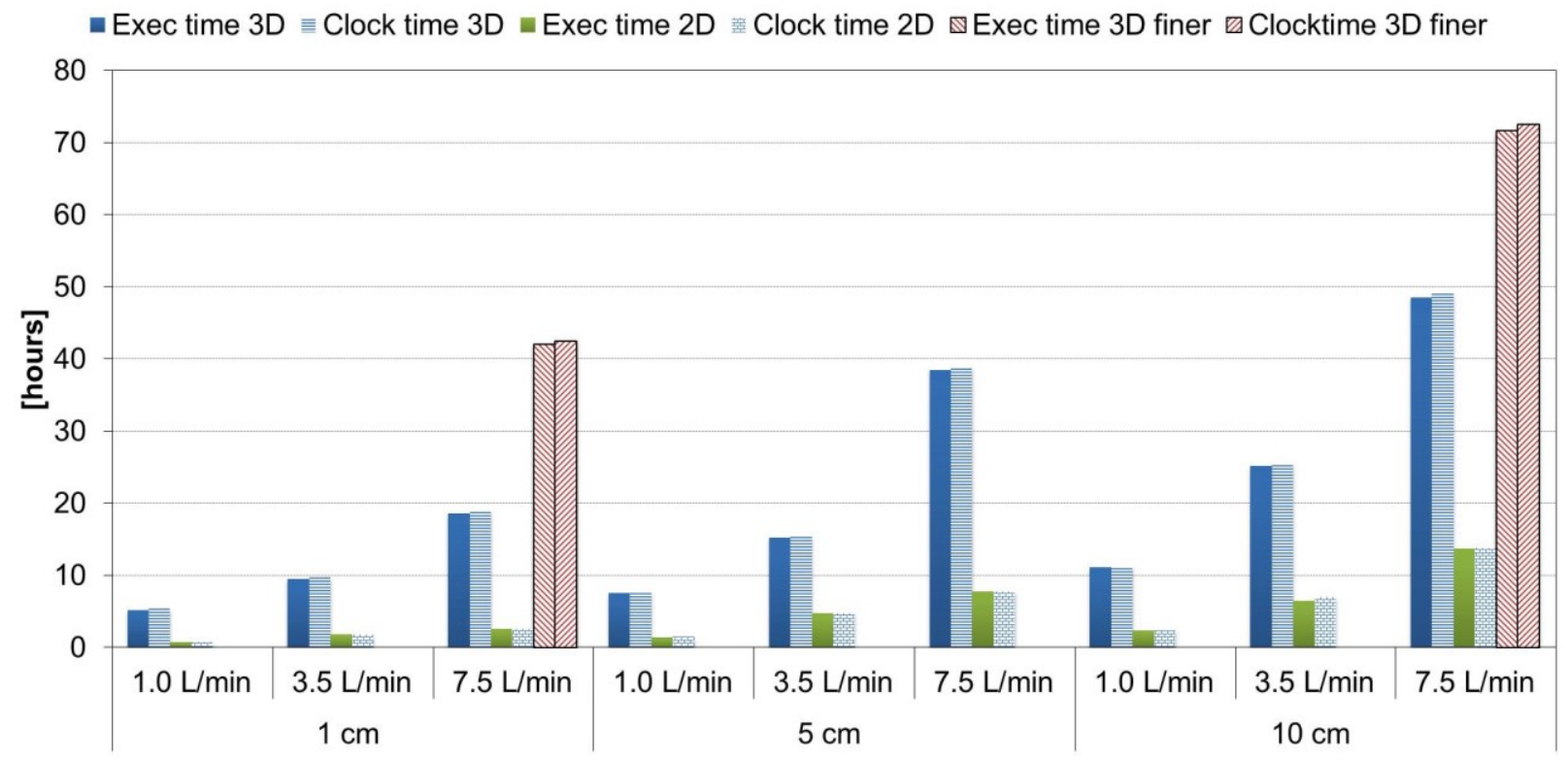

Figure 5: Cost of computation for 2D and 3D models $($ Exec time $=$ execution time of the code, Clock time $=$ execution time plus time to communicate between nodes and to write data to storage)

\subsubsection{Pressure signals}

Qualitatively, the pressure signals do show what is expected from moving from a twodimensional to a three-dimensional system, i.e., more subtle pressure fluctuations (see Figure 6). In the two-dimensional model, the reduced dimensionality appears to lead to physically unrealistic artifacts in the predicted pressure signal. This is evident when looking at the shape of the signal: the signal seems to decay from any peak, which is not expected from pressure signals. 

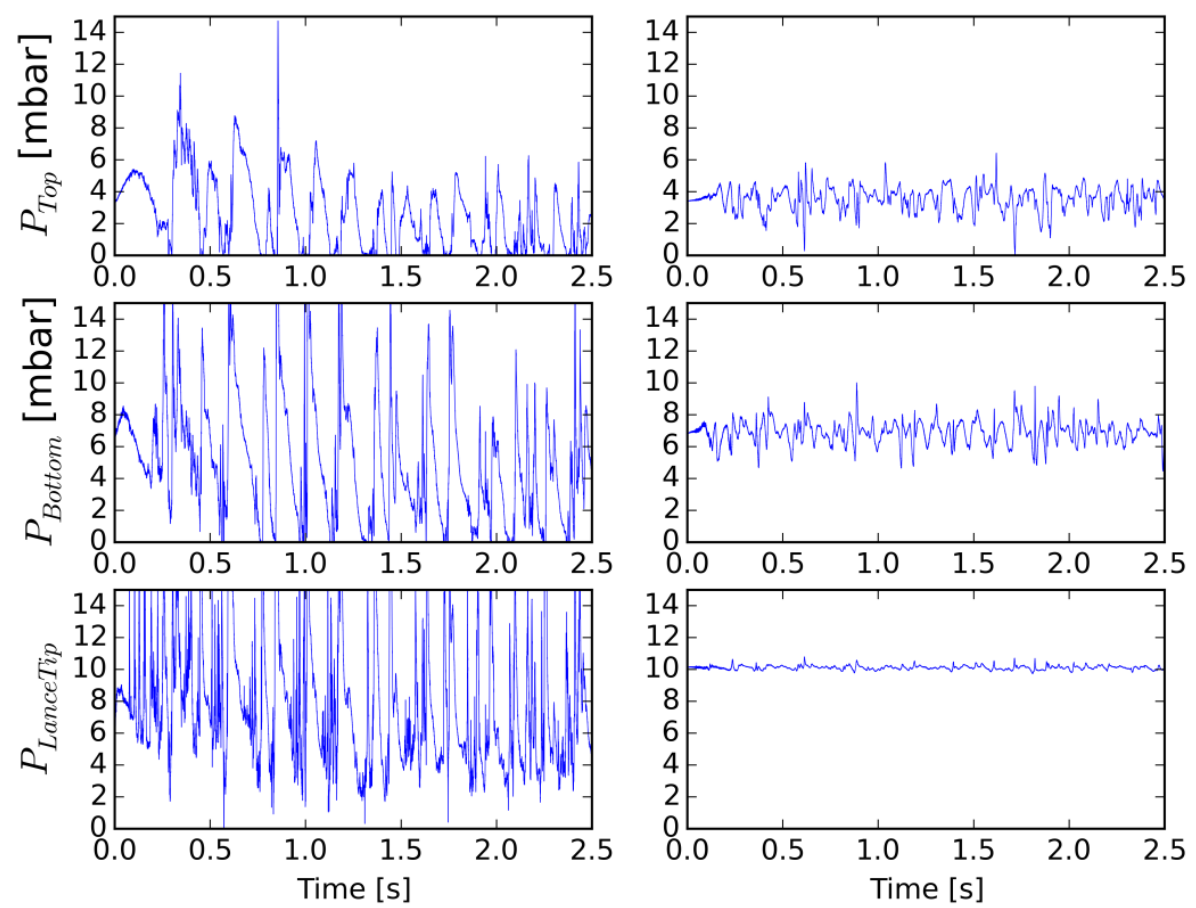

Figure 6: Pressure signal data from the two-dimensional (left column) and three-dimensional (right column) models. The pressure signal predicted for the top sensor, bottom sensor just above the lance (see Figure 2) and the lance tip is shown for a lance depth of $1 \mathrm{~cm}$ and a gas flow rate of $7.5 \mathrm{~L} / \mathrm{min}$. 
3.6.3. Visual comparison of the two computational models against experimental work
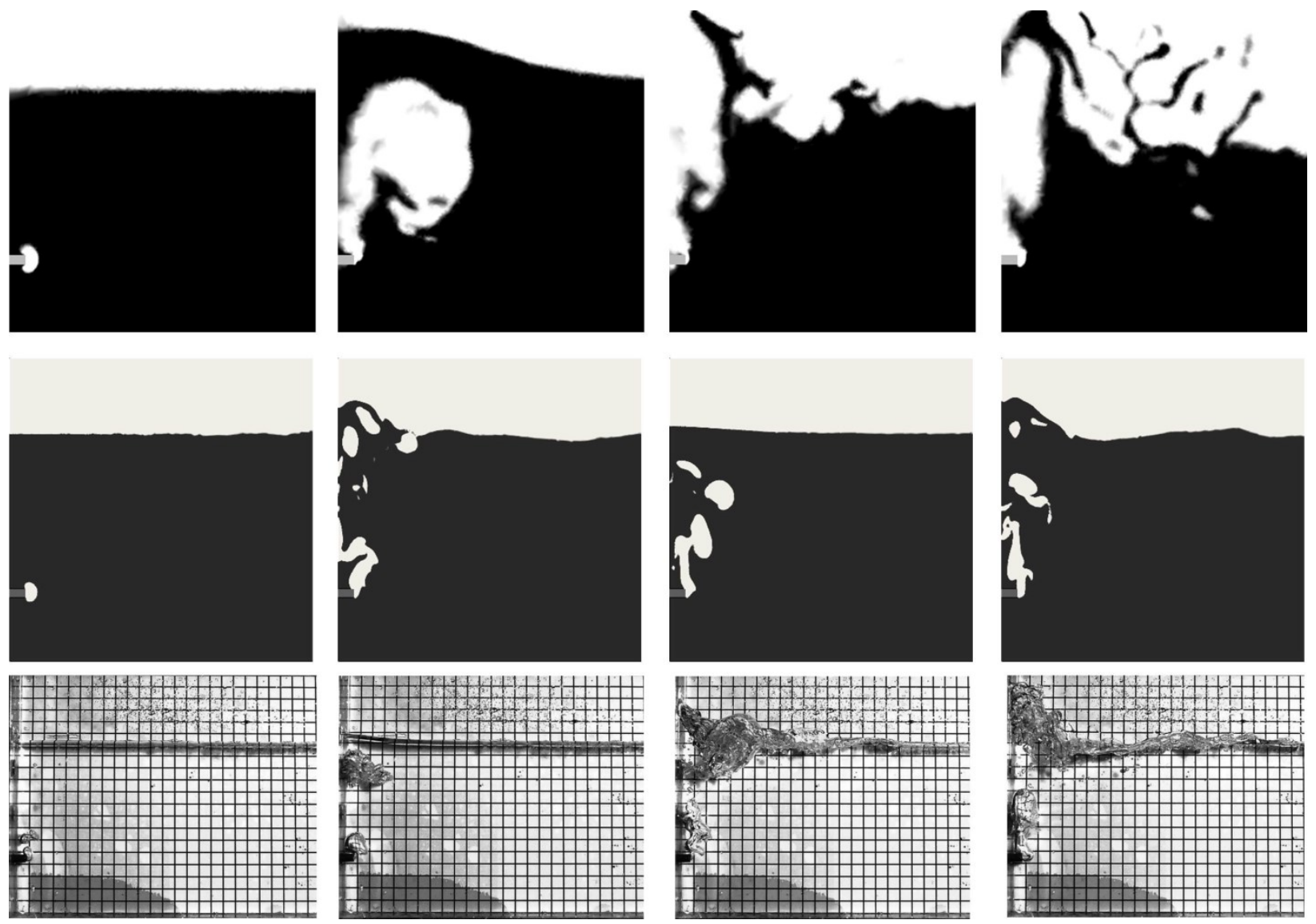

Figure 7: Comparison of the 2D (top row), 3D (middle row) and experimental (bottom row) model results. In the computational model results, the black phase is water $(\alpha=1)$, while the white/grey is air $(\alpha=0)$. The results are for a lance depth of $1 \mathrm{~cm}$, gas flow rate of $7.5 \mathrm{~L} / \mathrm{min}$. From left to right, these are still images at $\mathrm{t}=5 ; 205 ; 990$ and 1735 ms respectively.

Comparing the model results to experimental results from high-speed photography reveals significant differences:

1. At a time step of $205 \mathrm{~ms}$ (second column), the relative level of the water on the left wall is exaggerated in the $2 \mathrm{D}$ model compared to the $3 \mathrm{D}$ and physical model;

2. For $\mathrm{t}=990 \mathrm{~ms}$ (column 3) and $1735 \mathrm{~ms}$ (column 4), there is significant splashing and severe "tunneling" of gas against the wall of the tank (left), which is not present in the 3D or physical model;

3. At all time steps there are significant differences in the size and shape of the bubbles between the 2D model and the 3D and physical model.

It is, therefore, quite important that the three-dimensional models be developed further and the over-simplified 2D models be abandoned for the purposes of this study going forward. 


\section{Conclusions and current work}

A predictive model for oxygen lancing of tap-holes in pyrometallurgical furnaces can be useful in assessing the impact of the lancing process on both the tap-hole life and the process itself. In this work, a comparison was made between a two-dimensional and three-dimensional computational multiphase fluid flow model of a simple problem that relates to lancing. It was found that the three-dimensional model is physically more realistic, albeit computationally expensive. Current and future work in this area will involve expansion of the physical model to include multiple and more physically representative fluids in the experiments, as well as improving pressure signal measurement and analysis. Expansion of the three-dimensional model to include heat transfer and thermo-chemistry is also underway.

\section{Acknowledgements}

This paper is published by permission of Mintek. The authors would also like to thank the Centre for High Performance Computing (Cape Town) for making computing resources available for this project.

\section{References}

1. Nelson, L.R., and Hundermark, R.J. In Proc. SAIMM Furnace Tapping Conference, Muldersdrift, South Africa, 27-29 May 2014, pp. 1-32.

2. Nolet, I. In Proc. SAIMM Furnace Tapping Conference, Muldersdrift, South Africa, 27-29 May 2014, pp. 223-232.

3. Erwee, M.W., Reynolds, Q.G., Zietsman, J.H., Cromarty, R.D., and Lexmond, A.S. In Proc. INFACON XIV, Kyiv, Ukraine, 31 May - 4 June 2015, pp. 174-183.

4. http:// http://openfoam.org/ [Accessed 13 January 2016]

5. C. Geuzaine, and J.-F. Remacle. International Journal for Numerical Methods in Engineering 79(11), pp. 1309-1331, 2009.

6. Patankar, S. Numerical Heat Transfer and Fluid Flow. New York: Hemisphere Publishing Company (USA), 1980.

7. Hirt, C.W., and Nichols, B.D. Volume of fluid (VOF) method for the dynamics of free boundaries., Journal of Computational Physics, Vol. 39, 1981 pp. 201-225. 